\title{
Hemorrhagic Synovial Cyst Associated with Rheumatoid Atlantoaxial Subluxation
}

\author{
Jae Jon Sheen ${ }^{1}$, Dong Kwang Seo ${ }^{1}$, Seung Chul Rhim ${ }^{1}$, Seung Ho Choi ${ }^{2}$ \\ Departments of ${ }^{l}$ Neurological Surgery, ${ }^{2}$ Otolaryngotology, Asan Medical Center, \\ University of Ulsan College of Medicine, Seoul, Republic of Korea
}

Synovial cyst on prevertebral space of C1-2 joint is rare but may be associated hemorrhagic event. We describe a case of a 72-year-old woman who presented with sudden severe headache in her left occipital area with dyspnea. She had rheumatoid arthritis for 14-years. Large hemorrhagic cystic mass was seen around prevertebral space of the atlantoaxial joint on the left side on cervical MRI (magnetic resonance image) and it obstructed the nasopharyngeal cavity. Aspiration of the cystic lesion was performed via transoral approach, followed by posterior occipito-cervical fusion. The specimen was xanthochromic, suggesting old hemorrhage. The patient was tolerable on her postoperative course and showed good respiration and relieved headache. We suggest that repeated microtrauma due to atalantoaxial subluxation associated with rheumatoid arthritis as a main cause of hemorrhagic event on the cyst.

Key Words: Atlanto-Axial joint $\cdot$ Hemorrhage $\cdot$ Rheumatoid arthritis $\cdot$ Synovial cyst

\section{INTRODUCTION}

An atlanto-axial synovial cyst is very rare. Since the first report of this lesion by Onofrio and $\mathrm{Mih}^{11}$ in 1988, to our knowledge only 24 cases have been reported in the literature ${ }^{4,6,8)}$. The lesion often extends posteriorly to the dens and causes cervicomedullary compression ${ }^{4,6,8)}$. However, the current report described a patient with a large hemorrhagic cystic mass which was seen around prevertebral space of the atlantoaxial joint on the left side and the obstruction of the nasopharyngeal cavity on cervical magnetic resonance image (MRI).

We report a symptomatic case associated with rheumatoid atlanto-axial subluxation, which regressed after the surgical management.

\section{CASE REPORT}

A 72-year-old woman presented with sudden severe head-

- Received: February 4, 2013 - Revised: May 1, 2013

- Accepted: May 6, 2013

Corresponding Author: Seung Chul Rhim, MD

Department of Neurological Surgery, Asan Medical Center, University of Ulsan College of Medicine, 388-1 Pungnap-2dong, Songpa-gu, Seoul 138-736, Korea

Tel: +822-3010-3550, Fax: +82-2-476-6738

E-mail: gourmet1@naver.com ache in her left occipital area with dyspnea. The patient was alert and the function of cranial nerves was intact. Motor power and sensory were symmetric in all extremities. The dyspnea was aggravated on supine position. It was relieved on flexed head position. She had rheumatoid arthritis for 14-year, which was clinically and serologically proven. A laboratory examination of the patient's blood revealed no remarkable abnormality indicative of inflammation, but high-titer of rheumatoid factor $(95.9 \mathrm{IU} / \mathrm{mL})$. Seven months ago before visiting to our department, she checked brain MRI due to sudden attack of severe headache on left occipital area, which revealed non-specific lesion except rheumatoid pannus with small prevertebral cyst of C1-2 junction (Fig. 1).

On admission, there was a large hemorrhagic cystic mass around prevertebral space of the atlanto-axial joint on the left side, obstructing the nasopharyngeal cavity on cervical MRI (Fig. 2). The cystic mass was connected to atlanto-axial joint capsule on axial view of computed tomography (CT). Multiple bony erosion, rheumatoid atlanto-axial instability including left tilted C1-2 subluxation and cranial settling were demonstrated. And there were loss of lordosis and retrolisthesis in C 3-4, 4-5 (Fig. 3).

In the first operation stage, considering patient's dyspnea, aspiration of the cystic lesion was performed via transoral approach with otolaryngology surgeon (Fig. 4) and airway obstruction was relieved. The volume of aspirated cystic fluid was about $5 \mathrm{cc}$. The color of specimen was xanthochromic, 


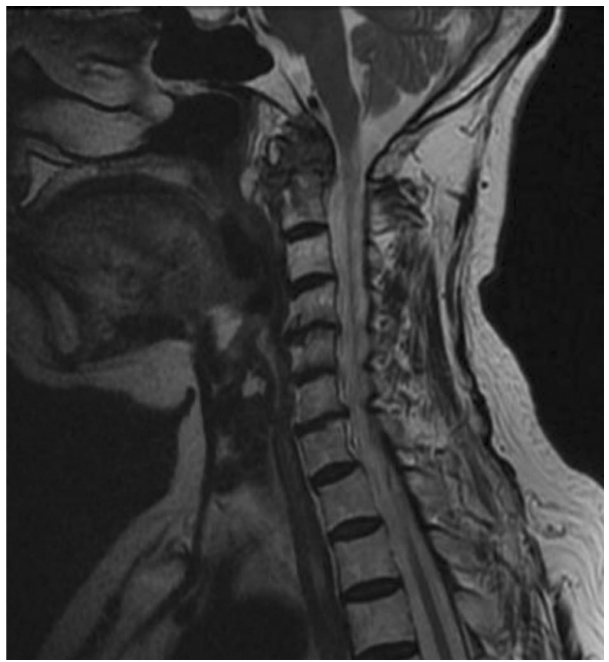

Fig. 1. The scout image of cenvical spine, included brain magnetic resonance imaging on previous visit: non-specific lesion except rheumatoid pannus formation with small prevertebral cyst of $\mathrm{Cl}-2$ junction.
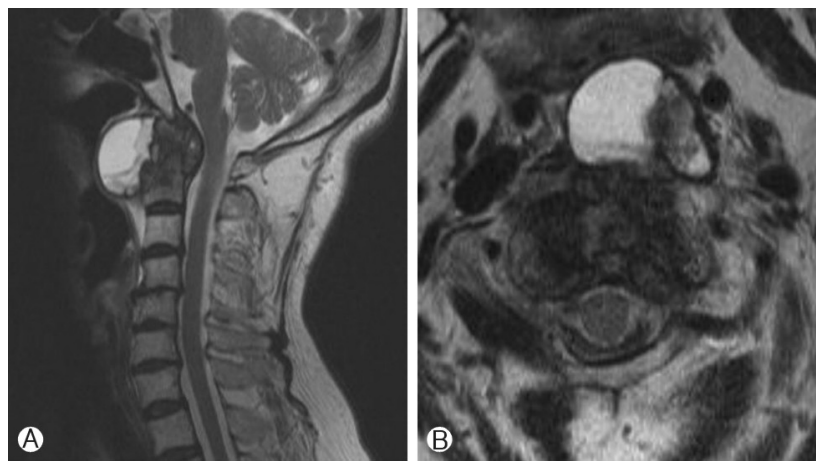

Fig. 2. A: Sagittal T2-weighted magnetic resonance imaging revealed rheumatoid atlanto-axial subluxation with pannus formation and hemorrhagic cyst at the prevertebral space and basilar invagination. B: Axial T2-weighted magnetic resonance imaging revealed rheumatoid atlanto-axial subluxation with pannus formation and hemorrhagic cyst at the prevertebral space and basilar invagination.

which suggested old hemorrhage. After $0.5 \mathrm{~cm}$-sized mucosal incision on left side oropharyngeal wall, residual material of the cyst was squeezed by forceps and removed by suction tools.

In the second operation stage, there were rheumatoid atlanto-axial instability and retrolisthesis in C 3-4, 4-5, so it was followed by posterior occipito-cervical fusion that connected from occiput to C5, using vertex screw \& rod system and iliac bone graft (Fig. 5). All the procedure was successfully done.

The patient was tolerable on her postoperative course and showed good respiration and relieved headache.

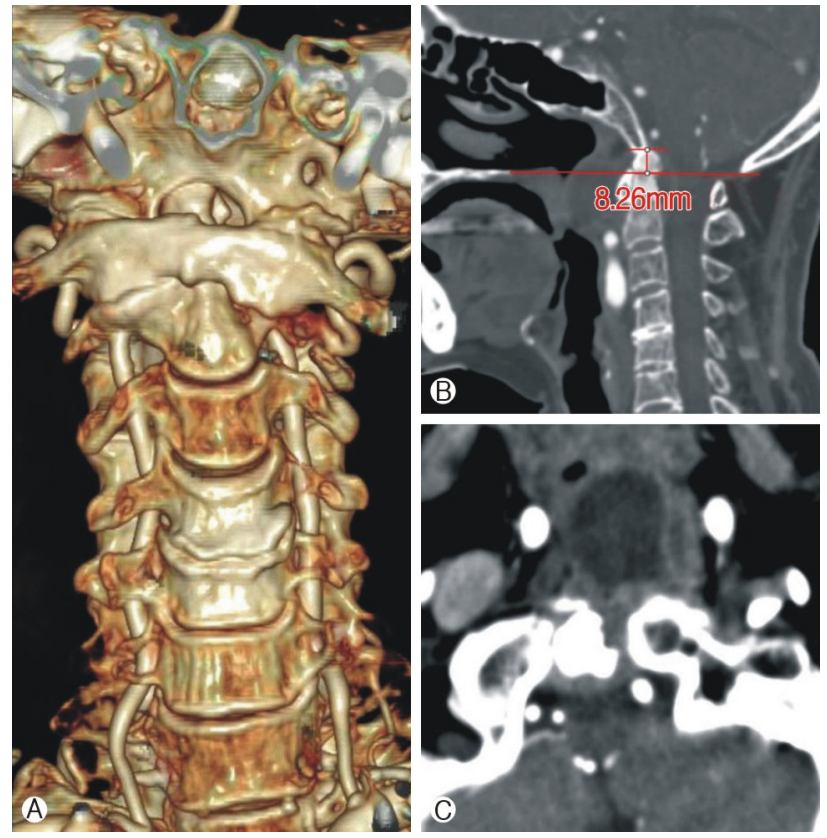

Fig. 3. A: Three-dimentional reconstruction of CT scan. B: Sagittal image of CT scan. C: Axial image demonstrated connection of cystic lesion and atlanto-axial joint cavity.
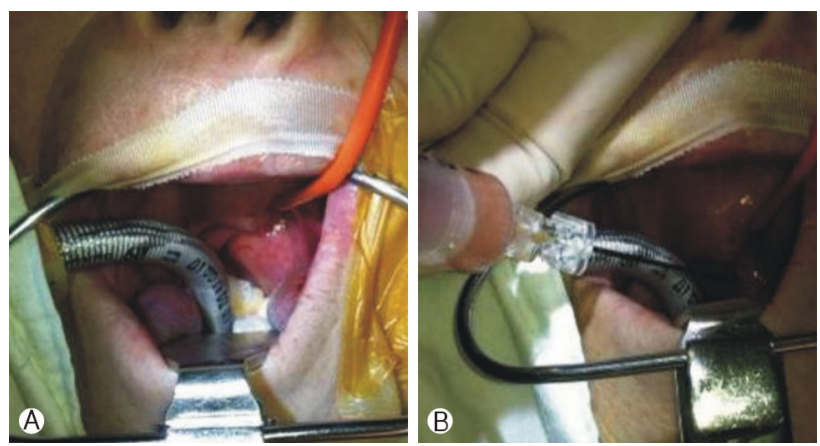

Fig. 4. A: The intraoperative photography demonstrated aspiration of cystic lesion via oral cavity. B: The intraoperative photography demonstrated aspiration of cystic lesion via oral cavity.

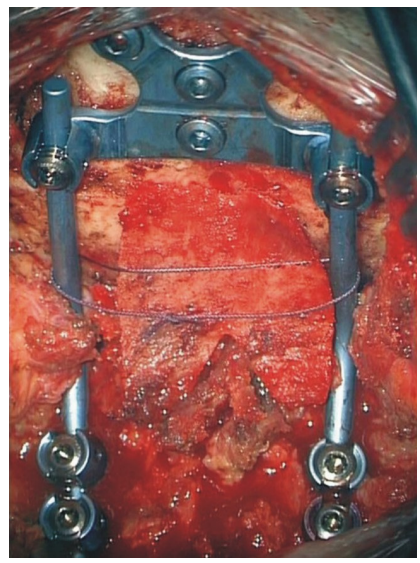

Fig. 5. The intraoperative photography demonstrated occipitocervical fusion. 


\section{DISCUSSION}

Cervical synovial cysts are rare and located at the C1-2 junction or lower cervical spine. Only 24 cases of synovial cysts of the C1-2 junction have been reported in the literature $^{5)}$. Moreover, hemorrhagic event of rheumatoid cysts in the C1-2 junction were not reported.

The pathogenesis of spinal synovial cysts remains unclear but is thought to be attributable to degenerative changes of the facet joints or excessive joint motion ${ }^{2,3,5,7)}$. The involvement of rheumatoid arthritis made the degenerative changes of joint more aggravate ${ }^{7}$. It revealed multiple bony erosions and a large pannus formation of C1-2 junction on this case.

This patient also showed atlanto-axial instability caused by rheumatoid arthritis, such as left tilted antlanto-axial subluxation and basilar impression. The atlantoaxial articulation is a true synovial joint and is responsible for a large proportion of normal cervical mobility. The etiology of articular cysts is unclear, but they are assumed to be degenerative because minor chronic damage to articular surfaces produces a reactive proliferation of synovium or fibrocartilage that includes loculated collections of mucinous fluid. Cysts may contain variable amounts of proteins, blood, or both ${ }^{2)}$. So, we hypothesized that hemorrhagic event was developed as a result of microtrauma caused by rheumatoid atlantoaxial instability.

It is known that the higher titer of rheumatoid factor, the more destructive manifestations of joint occur ${ }^{1)}$. The patient was in the controlled state in serologic inflammatory marker, such as ESR (Erythrocyte sedimentation rate) and CRP (C-reactive protein). However, the high titer of rheumatoid factor, in spite of long standing medication of rheumatology, was related factor of progressive destruction of joints.

\section{CONCLUSION}

We report a rare case of large hemorrhagic cyst on preverte- bral space of left side C1-2 area associated with rheumatoid arthritis causing airway obstruction and left occipital pain, successfully managed anterior and posterior approach. We suggest repeated microtrauma due to atlanto-axial subluxation associated with rheumatoid arthritis as a main cause of hemorrhagic event on the cyst.

\section{REFERENCES}

1. Aletaha D, Neogi T, Silman AJ, Funovits J, Felson DT, Bingham CO, $3^{\text {rd }}$, et al.: 2010 Rheumatoid arthritis classification criteria: an American College of Rheumatology/European League Against Rheumatism collaborative initiative. Arthritis Rheum 62(9):25692581, 2010

2. Birch BD, Khandji AG, McCormick PC: Atlantoaxial degenerative articular cysts. J Neurosurg 85(5):810-816, 1996

3. Chang H, Park JB, Kim KW: Synovial cyst of the transverse ligament of the atlas in a patient with os odontoideum and atlantoaxial instability. Spine (Phila Pa 1976) 25(6):741-744, 2000

4. Jabre A, Shahbabian S, Keller JT: Synovial cyst of the cervical spine. Neurosurgery 20(2):316-318, 1987

5. Kaufmann AM, Halliday WC, West M, Fewer D, Ross I: Periodontoid synovial cyst causing cervico-medullary compression. Can J Neurol Sci 23(3):227-230, 1996

6. Marbacher S, Lukes A, Vajtai I, Ozdoba C: Surgical approach for synovial cyst of the atlantoaxial joint: a case report and review of the literature. Spine (Phila Pa 1976) 34(15):E528-533, 2009

7. Morio Y, Yoshioka T, Nagashima H, Hagino H, Teshima R: Intraspinal synovial cyst communicating with the $\mathrm{C} 1-\mathrm{C} 2$ facet joints and subarachnoid space associated with rheumatoid atlantoaxial instability. Spine (Phila Pa 1976) 28(23):E492-495, 2003

8. Sagiuchi T, Shimizu S, Tanaka R, Tachibana S, Fujii K: Regression of an atlantoaxial degenerative articular cyst associated with subluxation during conservative treatment. Case report and review of the literature. J Neurosurg Spine 5(2):161-164, 2006 